\title{
Structural Study on Apoptosis of Chronic Eosinophilic Leukemia Cells by Interaction of S100A8 with Splicing Factor, Proline and Glutamine-Rich
}

\author{
Yubin Won ${ }^{1, \dagger}$, Hyosun $\mathrm{Choi}^{2, \dagger}$, In Sik Kim ${ }^{2,3}$, Ji Young Mun ${ }^{1,2, *}$ \\ ${ }^{1}$ Department of Biomedical Laboratory Science, College of Health Science, Eulji University, Seongnam 13135, Korea \\ ${ }^{2}$ BK21 Plus Program, Department of Senior Healthcare, Graduate School, Eulji University, Daejeon 34824, Korea \\ ${ }^{3}$ Department of Biomedical Laboratory Science, School of Medicine, Eulji University, Daejeon 34824, Korea
}

\begin{abstract}
${ }^{\dagger}$ These authors contributed equally to Chronic eosinophilic leukemia (CEL) is a myeloproliferative disease with an increased this work. number of mature eosinophils and their precursors, which results in infiltration into organs and organ enlargement. The main cause of this disease is the overexpression of tyrosine kinase. However, there is a need for alternative medication, because some patients are resistant to imatinib, which is a tyrosine kinase inhibitor for leukemia. Many studies have indicated that S100A8 and splicing factor proline and glutamine-rich (SFPQ) function as initiation signals of apoptosis in CEL cells. We reviewed structural studies on CEL cells related to S100A8 and SFPQ. Particularly, this review highlighted microscopic results for the study of S100A8 and SFPQ in CEL cells.

Received November 8, 2017
\end{abstract}

Revised December 10, 2017

Accepted December 15, 2017

Key Words: SFPQ, S100A8, Microscopy, Chronic leukemia

\section{INTRODUCTION}

Chronic eosinophilic leukemia (CEL) is a myeloproliferative disease involving the proliferation of mature eosinophils and their precursors. CEL is mostly caused due to FIP1-like-1 platelet-derived growth factor-alpha (FIP1L1-PDGFRa) fusion gene (Gotlib et al., 2004). Eosinophils include a variety of granulocytes, such as neutrophils and basophils, with granules and polymorphous nuclei; they account for approximately $1 \%$ to $5 \%$ of white blood cells $(<1,500 / \mu \mathrm{L})$ in normal individuals. The numbers of normal eosinophils and myeloblasts in peripheral blood are increased by $>1,500 / \mu \mathrm{L}$, $5 \%$ and 20\%, respectively (Gotlib, 2015). Especially, increased number of eosinophils can be found in the heart, central nervous system, lungs, and gastrointestinal tract, causing organ enlargement and infiltration into organs, such as the liver and spleen. The compound, imatinib for tyrosine kinase inhibition has been developed as the medication for leukemia (Kujak \& Kolesar, 2016), because the fundamental cause of leukemia, including CEL, is the overexpression of tyrosine kinase (Demoulin \& Montano-Almendras, 2012). Although imatinib is known to be an effective medication for CEL, the number of patients who show poor response to imatinib has been increasing due to resistance to imatinib (Falchi \& Verstovsek, 2015). A study on the differentiation, activation, and apoptosis of leukocytes is necessary, because there is no sufficient data regarding the diagnosis and therapeutic approach for CEL. For studying CEL, we summarized structural results related to apoptosis factors, such as S100A8 and splicing factor proline and glutamine-rich (SFPQ) for CEL cells. In patients with acute and chronic lymphocytic leukemia, the expression of S100A8 is elevated, and specially, the correlation between S100A8 expression and high-risk patients is confirmed by proteomic analysis (Asakura et al., 2016). Regulators of the binding between TLR4 or RAGE and S100A8 have been developed as new drugs for cancer (Maru et al., 2015). On the other hand, Narumi et al. (2015) reported S100A8 activates NK cells through RAGE signal pathway and

(a) This is an open-access article distributed under the terms of the Creative Commons Attribution Non-Commercial License (http://creativecommons.org/licenses/by-nc/4.0) which permits unrestricted noncommercial use, distribution, and reproduction in any medium, provided the original work is properly cited.

Copyrights @ 2017 by Korean Society of Microscopy 
inhibits tumors. Because the functions of S100A8 are not related to inhibition of tyrosine kinase activity, the regulation of S100A8 could also be effective in imatinib (inhibitor of tyrosine kinase) resistant cells. In addition, SPFQ is binding protein to S100A8 and is reported as a tumor suppressor (Dvinge et al., 2016). Interestingly, SFPQ was recently reported as redistributing to the cell membrane in malignant cell lines of leukemia patients, however the functional implications of this are not known (Ren et al., 2014). Therefore, we summarized structural results relating apoptosis factors such as S100A8 and SFPQ for the study of CEL.

\section{MAIN TEXT}

\section{S100A8}

S100A8 is a member of the $\mathrm{S} 100$ family of proteins, and is characterized by EF-hand $\mathrm{Ca}^{2+}$ binding protein, also known as myeloid-related protein (MRP)-8. S100A8 is abundant in myelocytes, such as neutrophils and eosinophils. It has been reported to play a role in apoptosis, chemotaxis, and inflammatory response through the extracellular signal pathways with toll-like receptor 4 (TLR4) and receptor for advanced glycation end products (Donato et al., 2013). It is a main factor not only in the inflammatory response but also in breast, prostate, and thyroid cancers. Recently, S100A8 has been reported to be related to proliferation and metastasis of tumors (Bresnick et al., 2015). Research on the relation of S100A8 to leukemia has been actively studying, because it is known that S100A8 is regulated in the differentiation process of HL-60, a human Caucasian promyelocytic leukemia cell (Asakura et al., 2016; Yang et al., 2016). Yang et al. (2012) reported that S100A8 can regulate early apoptosis in HL-60 cells by controlling the formation of microtubule-associated protein light chain 3 (LC3) which is an autophagy monitoring protein. Electron microscopy showed that on treatment with $\mathrm{As}_{2} \mathrm{O}_{3}$, the number of autophagosome-like vacuoles in HL-60 cells with S100A8 siRNA was lower than in cells with control siRNA (Fig. 1). It suggested that S100A8 is necessary for the

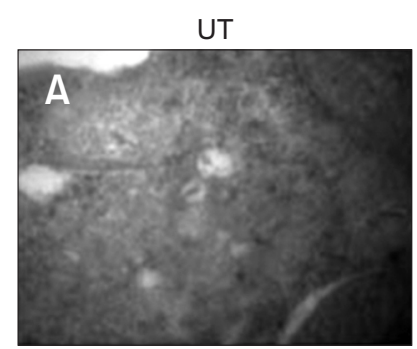

Control siRNA $+\mathrm{As}_{2} \mathrm{O}_{3}$

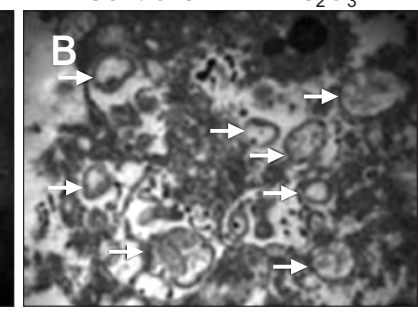

$\mathrm{S} 100 \mathrm{~A} 8 \mathrm{siRNA}+\mathrm{As}_{2} \mathrm{O}_{3}$

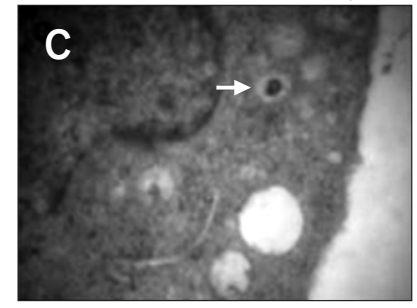

Fig. 1. Ultrastructural features in HL-60 cells with S100A8 siRNA after $\mathrm{As}_{2} \mathrm{O}_{3}$ treatment. On treatment with $\mathrm{As}_{2} \mathrm{O}_{3}$, the number of autophagosomes seen in cells treated with control siRNA $(5 \mu \mathrm{M}, 24$ hours) was higher than in cells treated with S100A8 siRNA. Arrows indicate autophagosomes; magnification, $\times 20,000$. UT, untreated group. Adapted from the article of Yang et al. (2012) (Int J Mol Med 29, 65-72) with original copyright holder's permission.
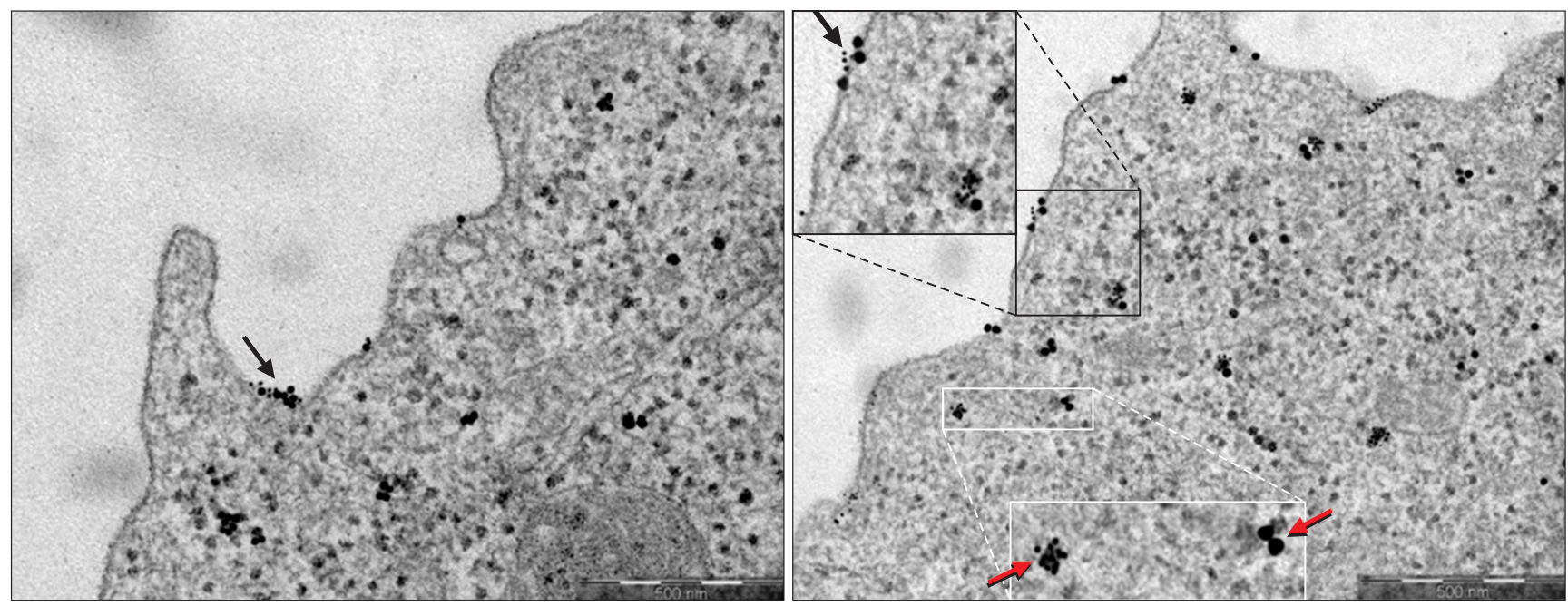

Fig. 2. The location of S100A8 and S100A9 proteins in THP-1 cells using immuno-gold transmission electron microscopy. Black and white boxes shows magnification of areas where S100A8 and S100A9 hetero- or homodimers can be observed, respectively. Black arrows represent the colocalization of S100A9 (large dots) and S100A8 (small dots) in cytoplasmic vesicles or the plasma membrane, and red arrows show vesicles containing only S100A9 or S100A8 homodimers. Adapted from the article of Chakraborty et al. (2015) (PLoS One 10, e0145217) with original copyright holder's permission. 


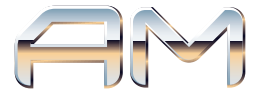

initiation of autophagy in leukemia cells. Yang et al. (2016) also showed that proautophagic activities were promoted by competitive inhibition of S100A8 for interaction between Beclin 1 and Bcl-2 by its binding to Beclin1. In addition, S100A8 increased the level of reactive oxygen species, which are generated in the mitochondria electron transport chain (mETC) complex, resulting in the induction of proapoptotic signals and engagement of the caspase cascade (Yang et al., 2012). Therefore, they suggested S100A8 controls the sensitivity of chemotherapy.

S100A8 (Fig. 2) is also located in cytoplasmic vesicles and plasma membrane as a heterodimer with S100A9 or as a homodimer in THP-1 cells and human leukemic monocytes (Chakraborty et al., 2015). S100A8 can modulate the function
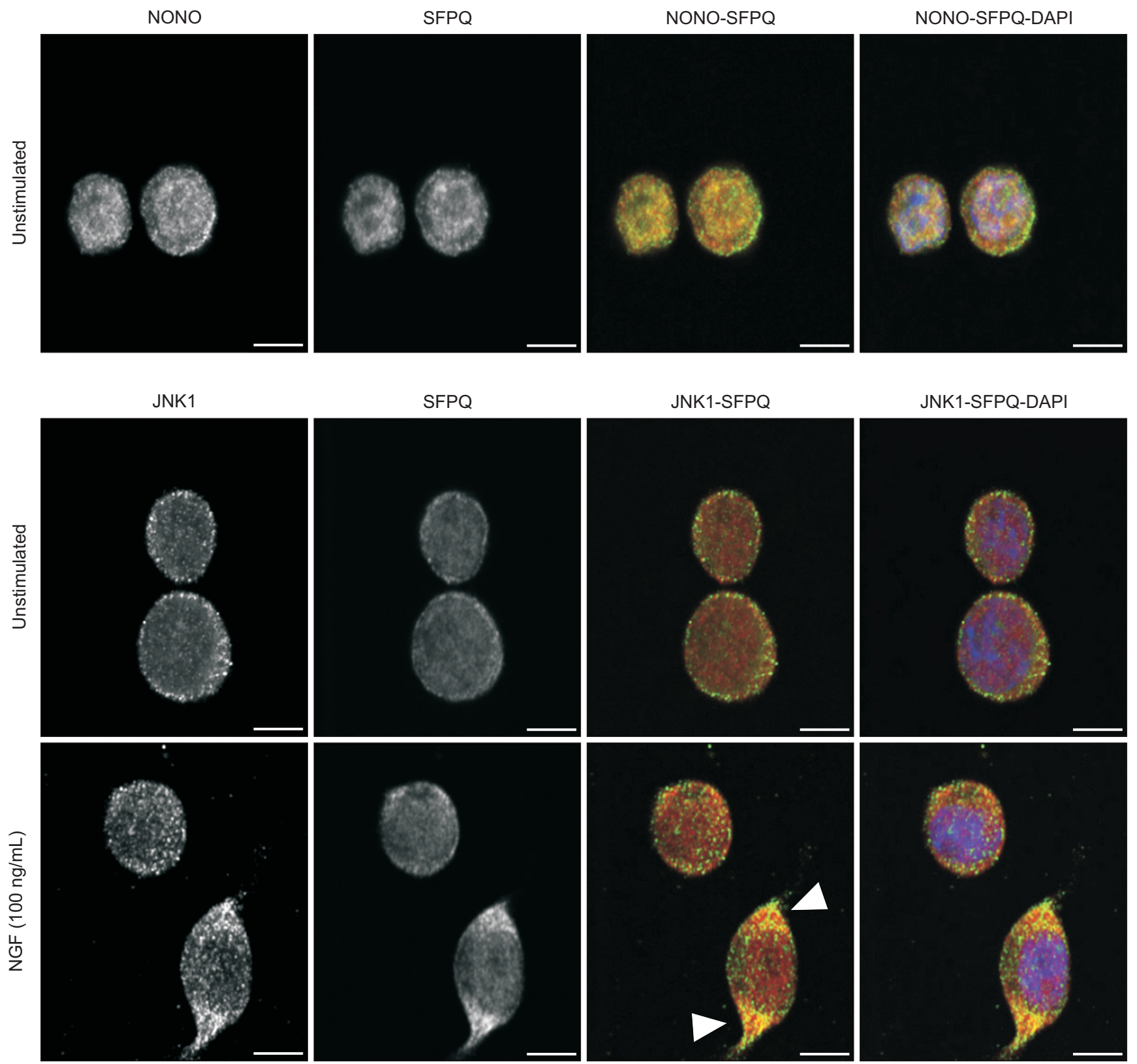

Fig. 3. Colocalization and cellular distribution of non-POU domain-containing octamer-binding protein (NONO), splicing factor proline and glutaminerich (SFPQ), and c-Jun N-terminal kinase (JNK). Confocal microscopy images of NONO (green), SFPQ (red), and nuclei (blue) in PC12 cells showed that NONO and SFPQ were expressed in the nucleus as well as cytosol. The colocalization of NONO and SFPQ mainly occurred in the nucleus without neuronal growth factor (NGF), and JNK was located in the cytosol. NGF treatment for 3 hours led to an increased colocalization signal of JNK1 (green; arrowheads) and SFPQ (red) in the cytosol. Scale bars=6 $\mu \mathrm{m}$. Adapted from the article of Sury et al. (2015) (Mol Cell Proteomics 14, 50-65) with permission from The American Society for Biochemistry and Molecular Biology. 
of other vesicle-associated proteins such as NADPH oxidase in these endocytic compartments (Kerkhoff et al., 2005). Further studies are still needed to clarify the particular function of S100A8 in cytoplasm.

In order to investigate how S100A8 regulate transcription factor, we reviewed tumor suppressor factor SFPQ, the binding protein to S100A8 in microscopic view.

\section{SFPQ}

In 1993, SFPQ was reported to bind polypyrimidine tract binding protein, and was named PTB-associated splicing factor because it is essential for pre-mRNA splicing (Patton et al., 1993). SFPQ has an important role in DNA repair, mRNA translation, and splicing. Furthermore, it binds PTB1, non-POU- domain-containing octamer-binding protein (NONO), and cell division cycle 5-like (CDC5L). In addition, SFPQ is involved in the pathogenic pathway of influenza virus and human immunodeficiency virus (LanderasBueno et al., 2011; Zolotukhin et al., 2003). Especially, SFPQ forms splicing factor-rich foci in a perinuclear pattern, related to the differentiation of myeloid cells (Shav-Tal et al., 2001). SFPQ-ABL1 fusion was found in B cell acute lymphoblastic leukemia, suggesting the association between SFPQ and leukemia; however, research on this topic has been limited. On the other hand, the structure of SFPQ on $\mathrm{X}$-ray crystallography showed a similarity to that of NONO including the DBHS family containing the DNA-binding domain, protein interaction domain (NOPS), and extended coiled coil domain. The alpha-helical region of $>110$ amino acids forms an unusual right-handed antiparallel coiled coil in the dimerization domain. Coiled coil structure is involved in the dimerization and interaction of DBHS proteins. Lee et al. (2015) showed that the localization of SFPQ in paraspeckles increased during extended SFPQ polymerization. Confocal laser microscopy also showed that residues 565 to 585 had a critical role in the localization of SFPQ in paraspeckles. Therefore, they suggested that the coiled coil interaction and polymerization domains of SFPQ are necessary for the localization in paraspeckles (Lee et al., 2015). The coiled coil structure was visualized using negative stain electron microscopy (Lee et al., 2015), and the location was investigated using confocal microscopy (Sury et al., 2015). SFPQ was accumulated in the dark nucleolar caps. After treatment with actinomycin D, a transcription inhibition drug, anxA10GFP strongly colocalized with SFPQ in MDCK cells. Paraspeckle component 1 (PSPC1), one of the three DBHS proteins, also colocalized with anxA10GFP in resting cells. This study indicated that colocalization and interaction between SFPQ and annexin 10A have an influence on the apoptosis (Quiskamp et al., 2014). Annexin 10A binding to SFPQ in the nucleus, and the overexpression of annexin $10 \mathrm{~A}$ is reported an inducing factor for tumor cell apoptosis. Annexin 10A localized in the nucleoplasm and cytosol, mainly in nucleoplasm of HeLa cell. Annexin 10A is recruited on the nucleolar or perinucleloar whose formation indicates that transcription inhibition is caused by apoptosis, mitosis, or RNA pol 2-inhibiting drug caps. The localization of SFPQ in the nucleus has been reported in various cell types. In addition, SFPQ was found in the cytosol when PC12 cells were differentiated using the neuronal growth factor (NGF). Sury et al. (2015) showed that SFPQ has a significant role in neuronal differentiation with interaction with c-Jun $\mathrm{N}$-terminal kinase (JNK) in the cytosol. The result can prove that SFPQ also has the function in cytosol as well as in nucleus. These results indicated that SFPQ functions in the cytosol as well as the nucleus. Western blot analyses showed that NONO and SFPQ were colocalized in the cytosolic and nuclear fractions, but more amount was shown in the nucleus than in the cytosol (Sury et al., 2015). The evaluation of proximity ligation assay signals with NONO antibody, SFPQ antibody, and 4',6-diamidino-2-phenylendole (DAPI) nuclear counterstaining also revealed that NONO-SFPQ colocalization was mainly found in the nucleus. Interestingly, NGF treatment induced the colocalization of SFPQ and JNK1 in the cytosol (Fig. 3). During polarization and outgrowth of primary neurites, SFPQ could be found in the cytosol. Then, SFPQ localizes only in the nucleus after the end of differentiation.

\section{SUMIMARY}

S100A8 elevated in leukemia patients, furthermore the level showed increase especially in drug resistance leukemia cell (Yang et al., 2014). In leukemia, SFPQ-encoding genes form a fusion protein that has a coiled-coil structure that promotes aberrant dimerization with partner proteins, and it can contribute to cancer (Dvinge et al., 2016). In leukemia, the increase of S100A8, the formation of SFPQ fusion protein, and the binding of the two proteins suggest us necessity of studying the two proteins. The mechanism of S100A8 inducing apoptosis in leukemia and the clarifying new intracellular functions of SFPQ could affect drug development for CEL.

\section{CONFLICT OF INTEREST}

No potential conflict of interest relevant to this article was reported.

\section{ACKNOWLEDGMENTS}

This paper was supported by Eulji University in 2016. 


\section{REFERENCES}

Asakura M, Karaki F, Fujii H, Atsuda K, Itoh T, and Fujiwara R (2016) Vildagliptin and its metabolite M20.7 induce the expression of S100A8 and S100A9 in human hepatoma HepG2 and leukemia HL60 cells. Sci Rep 6, 35633.

Bresnick A R, Weber D J, and Zimmer D B (2015) S100 proteins in cancer. Nat Rev Cancer 15, 96-109.

Chakraborty P, Bjork P, Kallberg E, Olsson A, Riva M, Morgelin M, Liberg D, Ivars F, and Leanderson T (2015) Vesicular location and transport of S100A8 and S100A9 proteins in monocytoid cells. PLoS One 10, e0145217.

Demoulin J B and Montano-Almendras C P (2012) Platelet-derived growth factors and their receptors in normal and malignant hematopoiesis. Am J Blood Res 2, 44-56.

Donato R, Cannon B R, Sorci G, Riuzzi F, Hsu K, Weber D J, and Geczy C L (2013) Functions of S100 proteins. Curr Mol Med 13, 24-57.

Dvinge H, Kim E, Abdel-Wahab O, and Bradley R K (2016) RNA splicing factors as oncoproteins and tumour suppressors. Nat Rev Cancer 16, 413-430.

Falchi L and Verstovsek S (2015) Eosinophilia in hematologic disorders. Immunol Allergy Clin North Am 35, 439-452.

Gotlib J (2015) World Health Organization-defined eosinophilic disorders: 2015 update on diagnosis, risk stratification, and management. Am J Hematol 90, 1077-1089.

Gotlib J, Cools J, Malone J M 3rd, Schrier S L, Gilliland D G, and Coutre S E (2004) The FIP1L1-PDGFRalpha fusion tyrosine kinase in hypereosinophilic syndrome and chronic eosinophilic leukemia: implications for diagnosis, classification, and management. Blood 103, 2879-2891.

Kerkhoff C, Nacken W, Benedyk M, Dagher M C, Sopalla C, and Doussiere $J(2005)$ The arachidonic acid-binding protein S100A8/A9 promotes NADPH oxidase activation by interaction with p67phox and Rac-2. FASEB J 19, 467-469.

Kujak C and Kolesar J M (2016) Treatment of chronic myelogenous leukemia. Am J Health Syst Pharm 73, 113-120.

Landeras-Bueno S, Jorba N, Perez-Cidoncha M, and Ortin J (2011) The splicing factor proline-glutamine rich (SFPQ/PSF) is involved in influenza virus transcription. PLoS Pathog 7, e1002397.

Lee M, Sadowska A, Bekere I, Ho D, Gully B S, Lu Y, lyer K S, Trewhella J, Fox A H, and Bond C S (2015) The structure of human SFPQ reveals a coiled-coil mediated polymer essential for functional aggregation in gene regulation. Nucleic Acids Res 43, 3826-3840.
Maru Y, Tomita T, Deguchi A, leguchi K, Takita M, Tsukahara F, Takemura K, Kitao A, and Gusovsky F (2015) Drug targeting based on a new concept-targeting against TLR4 as an example. Endocr Metab Immune Disord Drug Targets 15, 83-87.

Narumi K, Miyakawa R, Ueda R, Hashimoto H, Yamamoto Y, Yoshida T, and Aoki K (2015) Proinflammatory proteins S100A8/S100A9 activate NK cells via interaction with RAGE. J Immunol 194, 55395548.

Patton J G, Porro E B, Galceran J, Tempst P, and Nadal-Ginard B (1993) Cloning and characterization of PSF, a novel pre-mRNA splicing factor. Genes Dev 7, 393-406.

Quiskamp N, Poeter M, Raabe C A, Hohenester U M, Konig S, Gerke V, and Rescher U (2014) The tumor suppressor annexin A10 is a novel component of nuclear paraspeckles. Cell Mol Life Sci 71, 311-329.

Ren S, She M, Li M, Zhou Q, Liu R, Lu H, Yang C, and Xiong D (2014) The RNA/DNA-binding protein PSF relocates to cell membrane and contributes cells' sensitivity to antitumor drug, doxorubicin. Cytometry A 85, 231-241.

Shav-Tal Y, Lee B C, Bar-Haim S, Schori H, and Zipori D (2001) Reorganization of nuclear factors during myeloid differentiation. $J$ Cell Biochem 81, 379-392.

Sury M D, McShane E, Hernandez-Miranda L R, Birchmeier C, and Selbach M (2015) Quantitative proteomics reveals dynamic interaction of c-Jun N-terminal kinase (JNK) with RNA transport granule proteins splicing factor proline- and glutamine-rich (Sfpq) and non-POU domain-containing octamer-binding protein (Nono) during neuronal differentiation. Mol Cell Proteomics 14, 50-65.

Yang L, Yang M, Zhang H, Wang Z, Yu Y, Xie M, Zhao M, Liu L, and Cao L (2012) S100A8-targeting siRNA enhances arsenic trioxide-induced myeloid leukemia cell death by down-regulating autophagy. Int J Mol Med 29, 65-72.

Yang M, Zeng P, Kang R, Yu Y, Yang L, Tang D, and Cao L (2014) S100A8 contributes to drug resistance by promoting autophagy in leukemia cells. PLoS One 9, e97242.

Yang X Y, Zhang M Y, Zhou Q, Wu S Y, Zhao Y, Gu W Y, Pan J, Cen J N, Chen Z X, Guo W G, Chen C S, Yan W H, and Hu S Y (2016) High expression of S100A8 gene is associated with drug resistance to etoposide and poor prognosis in acute myeloid leukemia through influencing the apoptosis pathway. Onco Targets Ther 9, 4887-4899.

Zolotukhin A S, Michalowski D, Bear J, Smulevitch S V, Traish A M, Peng R, Patton J, Shatsky I N and Felber B K (2003) PSF acts through the human immunodeficiency virus type $1 \mathrm{mRNA}$ instability elements to regulate virus expression. Mol Cell Biol 23, 6618-6630. 